\title{
The Application of Probability Statistics in Higher Mathematics
}

\author{
Yugui Tang \\ Nanchang Institute of Science \& Technology
}

\begin{abstract}
Keywords: Higher mathematics; Probability theory; Inequation; Application
\end{abstract}
\begin{abstract}
Higher mathematics is a difficult subject and has many calculation and proving problems that are hard to solve without correct methods. However, if probability theory knowledge can be properly used, it cannot only improve the efficiency of problem solving, but also can help students develop the confidence to solve mathematical problems. Higher mathematics is a difficult subject. It has many calculation and proving problems that are hard to solve without correct methods. In solving such questions, if probability theory knowledge we can be properly used, it will improve answering efficiency and accuracy. This paper introduces the relative knowledge of probability theory, and discusses the probability theory in higher mathematics in details, as well as explains related issues of probability theory. It will provide reference for student to study the higher mathematics to understand the theory of probability in the application of higher mathematics.
\end{abstract}

\section{Introduction}

Probability theory is an important branch of higher mathematics and is widely used in daily life. It is a study on mathematical law of random phenomena. It originates in the middle of seventeenth century. At that time, mathematicians firstly thought of probability theory from the gambling problems. De mere, Pascal, Fermat, et al. studied and discussed on this problem firstly, then Bernoulli put forward the law of large numbers, Gaussian and Poisson further reasoned and proved the law. The development of society and the needs of engineering technical problems, prompted probability theory developing, and many scientists involved the study. Up to today, probability theory with the subjects of mathematical statistics based on it play an irreplaceable role in many fields, including natural sciences, social sciences, engineering and technology, military science and actual production and life and so on. So, the theory of probability is a very practical science, which deserves our attention.

The paper summarizes partial applications of probability theory in higher mathematics.

\section{Summary of Probability Theory}

Probability theory is a study on the occurring possibility of things, but the origin of initial probability theory associates with gambling problems. In the $16^{\text {th }}$ century, Italian scholar Girolamo Cardano began to study some simple problems in gambling, such as dice rolling.

The concepts and simple ways of probability and statistics are mainly used for gambling and demographic model. With human's social practice, people need to understand the implied inevitable regularity in all kinds of uncertain phenomenon, and study the possibility of various results using mathematics method, thus the theory of probability is created, and gradually developed into a rigorous discipline. Probability and statistics method is increasingly penetrated into all fields and widely used in natural science, economics, medicine, finance, insurance, and even the humanities.

With the development of science in 18th and 19th centuries, people noticed that in some biological, physical and social phenomenon, and there is some similarity with the game of chance, which makes the probability theory that originated from the game of chance be applied to these fields; at the same time it also greatly promoted the development of itself. The founder that made the theory of probability as a branch of mathematics is a Swiss mathematician $\mathrm{j}$. Bernoulli. He established the first limit theorem in probability theory, namely Bernoulli's law of large numbers, which clarifies the probability of event is stable at its frequency. Then Moivre and Laplace exported the basic primitive form of the second limit theorem (the central limit theorem). 
In the end of the 19th century, the Russian mathematician Chebyshev, Markov, Lyapunov et al. established the general form of the law of large numbers and central limit theorem with analysis methods, scientifically explained why many random variables encountered in actual life approximately obey the normal distribution. Under the stimulus of physics in the early 20th century, people started to study random process. Kolmogorov, Wiener, Markov, Wiener-Khinchin, Levy and Ferrer et al. made outstanding contributions.

Probability theory has been very mature up to today, and it's widely used in agricultural technology, military, engineering, biology, physics and other fields, improving the research level of the event occurrence, having laid a solid foundation for the development and progress of human society.

\section{Simplification with Probability Distribution}

Probability distribution is the basic concept in probability theory knowledge, and probability distribution properties can be used to simplify the problem solving process and improve the efficiency of problem solving. In solving the actual problems, the numbers between $0 \sim 1$ can be used to construct the occurring probability of certain events, ultimately achieving the goal of problem solving by means of the probability distribution knowledge. This kind of problem solving method can simplify the problem solving process, and improve the accuracy of calculation results, thus making the students understand the inner link between higher mathematics and probability theory more clearly, and cultivating students' interest in learning higher mathematics.

Eg. Calculation of $\sum_{k=2}^{a} \mathrm{C}_{a}^{k} x^{k} y^{a-k}(x>0, y>0)$

Solution: according to the uneven rules, toss a coin for A times, then the probability of right side up after the coin falling down is $\mathrm{P}=\mathrm{x} /(\mathrm{x}+\mathrm{y})$, use $\mathrm{T}$ to express the times of coin right side up, then:

$$
\mathrm{P}=\{\mathrm{T}=\mathrm{k}\}=C_{a}^{k} P^{k}(1-P)^{a-k},(k=0,1,2, \cdots a \text {. })
$$

According to the distribution law:

$$
\begin{aligned}
& \sum_{1=0}^{a} p\{T=k\}=\sum_{k=0}^{a} C_{a}^{k} P^{k}(1-P)^{a-k} \\
& =\sum_{k=0}^{\mathrm{a}} C_{a}^{k}(x / x+y)^{k}(y / x+y)^{n-k}
\end{aligned}
$$

At last, the calculated results of this question can be achieved smoothly:

$$
\sum_{k=2}^{a} \mathrm{C}_{\mathrm{a}}^{k} x^{k} y^{a-k}=(x+y)^{a}-y^{a}-a x y^{n-1}
$$

Besides, probability theory can be used to figure out the limit problems, eg.: Figure out the limit of $\lim _{a \rightarrow \infty} \frac{6^{a}}{a !}$. We can presumeלobeys the poisson's distribution of $\lambda=6$, then $(\xi=\mathrm{a})=\frac{6^{a}}{a !} e^{-6}, \sum_{\lambda=1}^{\infty} \frac{6^{a}}{a !}=1, \sum_{\lambda=1}^{\infty} \frac{6^{a}}{a !}=\mathrm{e}^{6}$ series, $\sum_{\lambda=1}^{\infty} \frac{6^{a}}{a !}=0$. For the more diffic
process.
Eg. $a_{x}\left[\frac{x}{1 !}+\frac{x^{2}}{2 !}+\frac{x^{3}}{3 !}+\cdots+\frac{x^{x}}{x !}\right] y^{-x}$ and based on the convergence necessity knowledge of point to solve this problem is to construct the probability model skillfully. Presume 
$\{\zeta \mathrm{i}\} \mathrm{i}=1,2,3,4 \ldots$ are independent sequence of random variables and obey the poisson's distribution of $\lambda=1, \quad \sum_{i-1}^{\mathrm{x}} \xi_{\mathrm{i}}$ will obey the Poisson's distribution of $\lambda=\mathrm{x}$, then

$$
\mathrm{P}\left(\sum_{i=1}^{x} \xi_{i} \leq x\right)=\sum_{k=0}^{x} P\left(\sum_{i=1}^{x} \xi_{i}=k\right)=\sum_{k=0}^{x} \frac{x^{k}}{k !} y^{-x}=\sum_{k=1}^{x} \frac{x^{k}}{k=1} y^{-x}+y^{-x}
$$

Also because $\mathrm{E} \zeta \mathrm{i}=\mathrm{D} \zeta \mathrm{i}=1$, based on the distribution central-limit theorem:

$$
\begin{aligned}
& \lim P\left(\sum_{i=1}^{x} \xi_{i} \leq x\right)=\lim _{x \rightarrow \infty} P\left[\frac{\sum_{i-1}^{x} \xi_{i}-x \times 1}{\sqrt{x \times 1}}\right] \leq \frac{x-x \times 1}{\sqrt{x \times 1}} \\
& \text { So: } \lim _{x \rightarrow \infty} a_{x}=\lim _{x \rightarrow \infty} a_{x} \sum_{k=0}^{x} \frac{x^{k}}{k !} y^{-x}=\lim \left[P\left(\sum_{i=1}^{x} \alpha \xi_{i} \leq x\right)-y^{-x}\right] \\
& =\lim _{x \rightarrow \infty} P\left(\sum_{i=1}^{x} \xi_{i} \leq x\right)-\lim y^{-x}=0.5-0=0.5
\end{aligned}
$$

\section{Proving of Inequations in Higher Mathematics}

In equation is also a quite difficult problem in higher mathematics. In the process of proof, the introduction of probability theory can greatly simplify the process, making complex problem simple.

Eg. Proving, if $\mathrm{b}=1,2,3 \ldots \mathrm{n} ; \mathrm{x}_{b} \geq 0$, then $\sqrt{\mathrm{x}_{1} x_{3} x_{3} \cdots x_{n}} \leq \frac{x_{1}+x_{2}+x_{3}+\cdots+x_{n}}{\mathrm{n}}$

$$
\ln \left(\prod_{b=1}^{n} x_{b}\right) \frac{1}{n}=\frac{1}{n} \sum_{b=1}^{n} / n b^{x b}
$$

. Proving: modeling first, let the distribution of random variable $\xi$ be $\mathrm{P}\left(\xi=x_{b}\right) \frac{1}{n}, b=1,2,3 \cdots n, \quad$ if $\mathrm{x}_{b}=0, \sqrt{\mathrm{x}_{1} x_{3} x_{3} \cdots x_{n}} \leq \frac{x_{1}+x_{2}+x_{3}+\cdots+x_{n}}{\mathrm{n}}$ exists, if $\mathrm{x}_{b}>0$, defining function $\mathrm{f}(\mathrm{a})=\operatorname{lna}(\mathrm{a}>0)$, then $\mathrm{f}(\mathrm{a})=\operatorname{lna}(\mathrm{a}>0)$ is up convex function, then from $f(\mathrm{E} \xi) \geq \operatorname{Ef}(\xi)$ :

$$
\mathrm{E}(f(a)) \leq f(E a)=\ln (E a)=\ln \left(\frac{1}{n} \sum_{b=1}^{n} x_{b}\right)
$$

Take e as the exponent of radix on both sides:

$$
\sqrt{\mathrm{x}_{1} x_{3} x_{3} \cdots x_{n}} \leq \frac{x_{1}+x_{2}+x_{3}+\cdots+x_{n}}{\mathrm{n}}
$$

\section{Figure out the Series and Improper Integral with Random Variable}

In probability theory, the digital characteristics owned by random variables are variance and mathematical expectation. The relationship between random variables and mathematical expectation can be effective for figuring out the series and generalized definite integral in higher mathematics.

Firstly, in higher mathematics, series is a kind of quite difficult calculation, if the relationship between the mathematical expectation and variance can be introduced into the process of calculation skillfully, hard things can be made simple and the problem can be solved successfully. 
Secondly, for the convenience of calculation, in the process of calculation, integrand can be transformed into the mathematical expectation of random variables under normal distribution case, and then calculating, so as to solve the problem.

Thirdly, for integral calculation, in the process of calculation, integration by parts can be used to solve, but in the process of calculation, it will be used repeatedly, and the limit should also be calculated, making the calculation quite complicated. To solve the problem, the mathematical expectation of exponential distribution random variables is often used for calculation so that you can successfully solve the problem.

Eg. Figure out the integrate of $\int_{0}^{\infty}\left(4 \mathrm{a}^{2}+5 a+6\right) \mathrm{b}^{-2 a} m a$

Solution: as the integrand has factor $\mathrm{b}^{-2 a}$, which meets the exponential distribution of $\lambda=2$, and the mathematical expectation is $\frac{1}{2}$, the variance is $\frac{1}{4}$, so:

$$
\begin{aligned}
& \int_{0}^{\infty}\left(4 a^{2}+5 a+6\right) b^{-2 a} m a=\frac{1}{2} \int_{0}^{\infty}\left(4 a^{2}+5 a+6\right) b^{-2 a} m a \\
& =\frac{1}{2} E\left(4 a^{2}+5 a+6\right)=2 E a^{2}+\frac{5}{2} E a^{2}+3 \\
& =2\left(\frac{2}{2^{2}}\right)+\frac{5}{2} \times \frac{1}{2}+3=\frac{21}{4}
\end{aligned}
$$

\section{Conclusion}

Many subjects in higher mathematics are difficult, and to decrease the difficulty, teachers should help students correctly understand the relative concepts of probability theory in teaching practice, and actively encourage and guide students to use knowledge of probability theory to analyze mathematical problems, to find a simple solving ways, thus improving the efficiency of problem solving, ensuring the correctness of calculation results, to help students set up their confidence in solving mathematics problems and gradually cultivate their interest in learning.

Enthusiasm of higher mathematics. In the calculation of higher mathematics, to use probability theory properly, teachers must guide the student to analyze corresponding concepts in probability theory, and make careful analysis against the concepts, grasping accurately definition type and the deformation of definition type, and then combining with higher mathematics calculation and proving for further careful calculation, which is the only way that the theory of probability and higher mathematics calculation are integrated organically, changing the complicated into simple, making the hard become easy, and solving the calculation problems in higher mathematics, and constantly improving the students' interest in learning higher mathematics.

\section{References}

[1] Marek-Crnjac L. The golden mean in the topology of four-manifolds, in conformal field theory, in the mathematical probability theory and in Cantorian space-time [J]. Chaos Solitons \& Fractals, 2006, 28(5):1113-1118.

[2] Eisen M. Introduction to mathematical probability theory [J]. Soobshcheniîa Akademii Nauk Gruzinskoî Ssr, 1968(4071):625.

[3] Fischer H. Dirichlet's contributions to mathematical probability theory [J]. Historia Mathematica, 1994, 21(1):39-63.

[4] Campos, Gerardo D, Campos, et al. The discovery of mathematical probability theory [electronic resource]: a case study in the logic of mathematical inquiry /[J]. 2005. 
[5] Gani J. Introduction to Mathematical Probability Theory by M. Eisen [J]. Revue De L Institut International De Statistique, 1971, 39(1):108-109.

[6] By Campos, Gerardo D. The discovery of mathematical probability theory a case study in the logic of mathematical inquiry /[J]. 2005.

[7] Watkins S J. Conviction by mathematical error? Doctors and lawyers should get probability theory right. [J]. Bmj Clinical Research, 2000, 320(7226):2-3.

[8] Pruitt W E, Pruitt W E. Review: Martin Eisen, Introduction to Mathematical Probability Theory [J]. Annals of Mathematical Statistics, 1971, 42(6):2159-2161.

[9] Hughes R D. Probability Theory and Mathematical Statistics for Engineers [M]// Probability theory and mathematical statistics for engineers /. Pergamon Press, 1984:973-974.

[10]Iosifescu M, Mihoc G, Theodorescu R. Probability Theory and Mathematical Statistics [M]// Probability theory and mathematical statistics:. World Scientific, 1996:333.

[11]Bikchentaev A M, Mushtari D K, Tikhonov O E. Department of Probability Theory and Mathematical Statistics, 1998-2002. [J]. At the Turn of - Centuries: 105-107.

[12] Shervashidze T L. Front Matter - Probability Theory and Mathematical Statistics for Engineers [J]. Theory of Probability \& Its Applications, 1983, 28(2):620вЂ““622. 